\title{
BMJ Open Coproduction of healthcare service with immigrant patients: protocol of a scoping review
}

\author{
Christina Radl-Karimi, ${ }^{1}$ Anne Nicolaisen, ${ }^{1}$ Morten Sodemann, ${ }^{2}$ Paul Batalden, ${ }^{3}$ \\ Christian von Plessen ${ }^{1}$
}

To cite: Radl-Karimi C, Nicolaisen A, Sodemann M, et al. Coproduction of healthcare service with immigrant patients: protocol of a scoping review. BMJ Open 2018;8:e019519. doi:10.1136/ bmjopen-2017-019519

- Prepublication history and additional material for this paper are available online. To view these files, please visit the journal online (http://dx.doi. org/10.1136/bmjopen-2017019519).

Received 7 September 2017 Revised 11 December 2017 Accepted 4 January 2018

Check for updates

${ }^{1}$ Centre for Quality, Region of Southern Denmark, Middelfart, Denmark

${ }^{2}$ Migrant Health Clinic, Department of Infectious Diseases Q, Odense University Hospital, Odense, Denmark

${ }^{3}$ The Dartmouth Institute for Health Policy and Clinical Practice, Dartmouth College, Lebanon, New Hampshire, USA

Correspondence to Christina Radl-Karimi; crk@rsyd.dk

\section{ABSTRACT}

Introduction Immigrant patients often meet barriers to patient-centred healthcare in their new host countries. Given the heterogeneity of patients from ethnic minorities, established strategies for patient centredness might not work in their case. The concept of coproduction provides a new perspective on how to collaboratively create the highest possible value for both the patient and the healthcare system. The concept acknowledges that all service is coproduced and directs attention to the relationship between patient and care provider. Coproduction is still a new concept in healthcare and its use with vulnerable groups of patients requires further study. This protocol outlines a scoping review to be conducted on the current knowledge on coproduction of service by immigrants and their service providers in the healthcare sector.

Methods and analysis We will use Joanna Briggs methodology for scoping reviews. The data will stem from the following databases: PubMed, Scopus, Ovid EMBASE, EBSCO CINAHL, EBSCO PsycINFO, Cochrane Library and Web of Science. We will also screen the websites of national authorities and research organisations for publications and review the literature lists of the identified articles for relevant references. We will include all types of literature on coproduction of healthcare or social service by immigrants and service providers, including their relationship with one another, communication and collaboration. Two reviewers will independently screen eligible publications and extract data using a checklist developed for this scoping review.

Ethics and dissemination The results of the study will provide an innovative perspective on the coproduction of value in healthcare service by immigrant patients and care providers. We will present the results at national and international conferences, seminars and other events with relevant stakeholders and immigrant patients, and publish them in a peer-reviewed journal.

\section{INTRODUCTION}

Immigrants often experience barriers when accessing healthcare service in primary and secondary medical facilities in their host country. These barriers, often caused by language and cultural differences, lack of social support or challenges related to transportation or employment put them at risk

\section{Strengths and limitations of this study}

- The literature on coproduction with immigrant citizens/patients has not been mapped previously. The review will provide valuable insights into the current knowledge on coproduction of service between immigrants and their service providers in both the healthcare and the social/community service sectors.

- Our broad search strategy goes beyond the term 'coproduction,' capturing aspects of coproduction in similar concepts of relationships between patients from ethnic minorities and providers, for example, patient/community involvement and cross-cultural communication.

- We will pay special attention to factors fostering capability for coproduction.

- The scoping review will be conducted in accordance with established guidelines. Two reviewers will independently screen the literature and read the full text.

- The broad search strategy incurs the risk of a wide spectrum of disparate results, which can be challenging to overview.

for coproducing and receiving suboptimal care. $^{12}$ However, suboptimal care can also occur because of unintentional provider behaviour. Even care providers who are motivated to be non-prejudiced may stereotype immigrant patients because they struggle with the great diversity of the patient group. $^{3-5}$ Immigrant patients do not only differ from the main population; they are also a heterogeneous group themselves. They differ by ethnicity, culture, religion and their reason for migration. ${ }^{6}$ This complex mixture of cultures and backgrounds makes it even more challenging for them to develop and to be capable of coproducing a service, to fit in and to have their health and welfare care needs met. ${ }^{7}$

Based on the growing belief that involving patients can improve the quality of care, ${ }^{89}$ over the past decade, healthcare systems have 
been infused with innovative strategies for shared decision-making, and patient centredness and participation. ${ }^{10} 11$ These approaches might be beneficial for patients who actively participate in the medical consultation by expressing their concerns, asking questions and stating their expectations. However, other patient groups (including, for instance, immigrant patients) are not only less inclined to take an active role in the consultation; they also may be less likely to have their involvement supported by the healthcare professional. ${ }^{12}$ Thus, even within strategies of patient centredness, the patient still depends to a certain degree on the care provider, ${ }^{13}$ which might limit their full effectiveness to improve patient outcomes. ${ }^{14}$ It has been suggested that such predefined and standardised approaches to the provision of healthcare service resemble the logic for making a product, rather than a service. Therefore, this confusion may contribute to the slow progress of services that are truly patient centred. ${ }^{15}$ This suggests that a fresh frame for exploring the relationship between patient and care provider may offer new insights into how healthcare service can create the best possible value contribution for the health of all patients, and especially marginalised groups such as immigrant patients.

\section{Coproduction of healthcare service}

The concept of coproduction, as a new perspective, has great potential to improve healthcare service delivery. Originally established in the 1970s by political economist Elinor Ostrom, ${ }^{16}$ coproduction has only recently been introduced to healthcare but is quickly gaining momentum, both in practice and in research. ${ }^{15} 1718$ According to Batalden, ${ }^{19}$ coproduction in healthcare is "the interdependent work of patients (and relatives) and health care professionals to design, create, develop, deliver, assess, and improve relationships and actions that contribute to the health of individuals and populations." Thus, the core of healthcare service provision lies in the individual relationship between patient and care provider-a relationship in which the coproducers both contribute resources and benefit from the value created by the service provided. The value created for patients comprises, for instance, their satisfaction with the service, the impact of the service on their well-being and the extent to which it meets their social, health or economic needs. Service coproduction does also create 'public value' by contributing to societal objectives or well-being. ${ }^{20}$

Based on Osborne's conceptualisation of the topic, ${ }^{20}$ our understanding of coproduction comprises (1) the 'pure' coproduction, in which the user unavoidably coproduces the service experience and outcomes with a service provider and (2) how the service experience integrates with the user's overall life experience. In healthcare, this includes both the direct encounter between patient and care provider and how the experience of the coproduced service integrates with the patient's overall life experience. This implies sharing values and interdependence between patient and professional. It involves letting patient and family priorities influence the civil discourse when planning the implementation of healthcare service. $^{15}$

Coproduction is present in any encounter between patient and professional intent on developing and creating a service. The degree and form of coproduction can vary across time, setting and circumstance. In addition, patients and care providers have widely disparate coproduction dispositions and capacities. ${ }^{15}$ Despite the overall optimism around the concept, there are also critical voices emphasising that coproduction can not only empower but also exploit patients. Constant cost-constraint pressures and a reluctance to release power are playing a role in the providers' inability to coproduce. ${ }^{21}$ Moreover, service providers need to be able to facilitate and create relationships, be adaptable and act as a link between citizen and system in order for coproduction to happen. ${ }^{22}{ }^{23}$ Moreover, disadvantaged citizens (eg, immigrants) may be constrained by a lack of knowledge or other resources necessary to contribute to and benefit from a coproduction process. However, if coproduction strategies are designed to lift the underlying constraints of disadvantaged service users (eg, lack of knowledge or resources), they may increase both efficiency and equity in the service delivery. ${ }^{24}$

In recent years, a multitude of coproduction efforts between the public sector and the civil society via community-based interventions have been established in Denmark. ${ }^{25-27}$ Yet, there is still little experience with the concept in healthcare service. This calls for further investigations on coproduction under varying conditions and testing whether the experiences collected from community based interventions can be transferred to the healthcare sector. The additional focus on immigrants can render valuable insights on how to improve the quality of their care and eventually contribute to better health. To that end, this article outlines a protocol for a scoping review on the current knowledge on coproduction of service between immigrants and their service providers in the healthcare and social/community sectors.

\section{STUDY DESIGN AND METHODS}

The scoping review methodology is particularly useful for systematically examining broad areas of evidence from disparate and heterogeneous sources and identifying key concepts, theories, evidence or research gaps. ${ }^{28} 29$ Thus, the scoping review method fits our purpose of providing a broad overview of the existing published and unpublished literature on service coproduction between immigrants and service providers. Unlike systematic reviews, scoping reviews do not focus on the effectiveness of a specific intervention but are used to map key concepts of a certain research area or to clarify the conceptual boundaries of a topic. Moreover, a scoping review allows for ongoing reflections, potentially considering emerging evidence and ongoing adjustments to the search strategy. The scoping review will be conducted according to the 
methodology proposed by the Joanna Briggs Institute, ${ }^{30}$ which is based on the five-stage framework laid out by Arksey \& O'Malley ${ }^{28}$ and Levac et $a l^{29}$

\section{Stage 1: identifying the research question}

The following research questions will guide the development of the protocol, facilitate the literature search and provide a structure for the scoping review report:

- What are the individual and context-related factors influencing the coproduction of value in healthcare between immigrant patients and their care providers?

- How do these individual and context-related factors affect the coproduction process between immigrant patients and their care providers?

- What learnings on coproduction of value for immigrant citizens in the community sector may be applied to coproduction in healthcare?

Coproduced healthcare service by immigrants and service providers can be influenced by a variety of individual and context-related factors. Individual factors can influence the capability to coproduce and can relate to a member of the dyadic, interdependent relationship. Patient-related factors include sociodemographic backgrounds, previous expectations of and experiences with the healthcare system or their capacities and attitudes toward involvement. Care provider-related factors on the other side can for instance relate to the care providers' preparedness and their understanding of immigrant health needs, as well as their attitudes or behaviours towards the immigrant patient. Context-related factors can be of either an objective or a subjective nature. ${ }^{31}$ They can include tangible (objective) factors such as the organisation of an integrated healthcare system, clinical guidelines or even the clinical surrounding. In contrast, the subjective context focuses on how patients and care providers interpret and attach significance to what is happening around them and how that influences their own behaviour and interaction with one another.

The literature search will cover two potential arenas of coproduction: (1) between immigrant patients and care providers in the healthcare sector and (2) between immigrant citizens and social service providers in the community sector. In recent years, Denmark has seen an increasing interest in developing new ways of establishing collaborations between citizens and service providers in the production and delivery of welfare benefits. ${ }^{25}$ In healthcare, coproduction is usually of a involuntary nature because patients have to coproduce if they want better health. On the community level, coproduction is more of a conscious and voluntary act and is, for instance, concerned with how to empower citizens or improve overall service delivery. ${ }^{20}$ However, we decided to include the community sector to investigate whether we can learn from coproduction experiences and see if these findings can be applied within a healthcare context.

\section{Stage 2: identifying relevant studies}

Our preliminary search strategy was developed in consultation with a medical librarian at the University of Southern Denmark (see online supplementary appendix 1). Elements of coproduction can also be found in other concepts such as patient/citizen involvement and participation, shared decision-making and patient/ citizen centredness and empowerment. Therefore, these concepts will be included in the search strategy of literature from the health and social sciences, namely sociology, anthropology and psychology.

\section{Peer-reviewed literature}

We will conduct a systematic search of peer-reviewed literature using a three step search strategy in licensed journal databases, including all study designs and methodology. The first step is an initial limited search in the PubMed and Scopus databases relevant to the topic. This step has already been undertaken on 16 August 2017 and yielded 1018 hits in the PubMed and 159 in the Scopus databases. In the second step, we will use all identified keywords and index terms from the initial search and translate them in Ovid EMBASE, EBSCO CINAHL, EBSCO PsycINFO, Cochrane Library and Web of Science databases. In the third step, we will search the reference lists of the identified relevant articles for additional studies. Fulltext publications in English, Scandinavian languages (Danish, Swedish and Norwegian) and German will be considered for inclusion, because the authors can read these languages. No geographic limits will be used for the peer-reviewed literature search, since we expect the principal concept of coproduction to be comparable across countries. The search will be restricted to publications from 2007 onwards when patient-centred care was beginning to take root and appear in medical literature. $^{32}$ We will use EndNote to remove duplicates and store bibliographic information.

\section{Non-peer-reviewed literature}

We will also screen non-peer-reviewed literature to identify non-indexed reports, government documents, guidelines, policy papers and dissertations. We will search websites of Danish national authorities, research institutions and other relevant interest organisations. To gather comparable publications from another national setting - without moving beyond the feasible scope of this review-we will also search corresponding websites in the UK. This country was chosen because of its comprehensive experiences with the coproduction concept in healthcare. ${ }^{33}$

\section{Stage 3: study selection}

The PCC (participant, concept, context) mnemonic suggested by the Joanna Briggs Institute ${ }^{30}$ provides a transparent guide for reviewers and readers and will direct the decision process on which sources to include in the scoping review. 


\section{(P) Participants}

In the scoping review, we will focus on the coproductive relationship between immigrant patients/citizens and care/service providers. Therefore, both sides of this relationship will be included as participants. The search will include literature on immigrants of any origin, age or sex. Immigrants are defined as foreign born people who have moved to another country for the purpose of settlement. ${ }^{34}$ This definition includes economic migrants, temporary foreign workers, foreign students, documented and undocumented migrants, refugees and asylum seekers. We will also include literature on descendants (ie, neither of the parents was born in the country they live in) because they tend to resemble first-generation immigrants when it comes to morbidity and self-perceived health. ${ }^{35}$ To get a more inclusive view of the evidence on the patient/citizen target group, we decided also to include ethnic minorities in the search. Searching only for immigrants might result in too narrow findings and useful insights relevant for immigrants can be embedded in publications on ethnic minorities. We define ethnic minorities as a group within a community whose national or cultural traditions differ from those of the main population. ${ }^{37}$ This includes immigrants, their descendants and groups of people who were born in a certain country but still count as a minority (such as Hispanics, Native Americans and Aborigines). On the service provider side, we will include all types of health professionals and social service providers that are delivering personal service for ethnic minorities. This can include service providers from the public/state or the voluntary/non-profit sectors that work on social, health or educational activities for ethnic minorities on a community level. Literature with researchers as participants on the provider side will be accepted, if the participating ethnic minority target group has been coproducing participatory research instead of merely being consulted on a certain research topic.

\section{(C) Concept}

In this scoping review we, will analyse coproduction as it happens in the joint activity between immigrants and their service providers. This can happen either through ongoing personal interaction in which both parties perform most of the task together (eg, in a patient-physician consultation) or through processes in which citizens act separately for most of the time and only deal with the service provider at particular points, when the gains of their efforts are combined (eg, in between consultation appointments). ${ }^{38}$ This includes face-to-face encounters in consultations as well as group activities such as shared medical appointments, in which immigrants coproduce strategies with their peers. Additionally, we will include publications on the relationship, communication or collaboration between immigrants and service providers because they are strongly related to the concept of coproduction and might entail coproductive elements. We will use a broad definition of communication, including verbal or non-verbal behaviour, interaction and interpersonal knowledge, skills and habits. Publications on community-based participatory research will be included if immigrants (1) have been actively coproducing the research and (2) benefit from the value created by the research project. Framing the concept of coproduction this way will allow us to include literature with an intention to coproduce service between immigrants and service providers, as well as publications with an unexpected, but retrospectively recognised, coproduced outcome.

\section{(C) Context}

We will include two different arenas in which coproduction by immigrants and care/service providers can occur: in healthcare and community settings. By healthcare, we mean the primary and secondary healthcare sector. Examples in primary healthcare are general practitioners, specialists, pharmacies, home care, nursing homes and community nurses. In the secondary healthcare sector, public, private, somatic and psychiatric hospitals will be included. All types of healthcare service available for patients in ambulatory care, day care, long-term care and social care will also be included. The second arena includes community settings in which individual immigrant citizens or communities actively participate in delivering social service. The definition of social services used here is: (1) they are personal services, rather than services related to the production of goods; (2) they fulfil personal social rather than physical or intellectual needs; (3) they focus on social roles rather than bodies, intellects or minds, thus distinguishing them from health, education and psychological assistance and (4) they are performed person to person in direct social interactions. ${ }^{39}$

Citations will be screened by using the web-based software Covidence (www.covidence.org). Covidence also facilitates the creation of a PRISMA flow diagram once the screening process is completed. Two reviewers (CR-K and AN) will screen titles and abstracts against the PCC criteria and mark them 'include,' 'exclude,' 'uncertain' or 'relevant for other purposes.' A summary of all inclusion and exclusion criteria in regard to form and content is shown in box 1 . To ensure reliability between the reviewers, the inclusion/exclusion criteria will be pilot tested on a random sample of citations. If no abstract can be identified, the publication will be dismissed. The reviewers will discuss uncertainties or differences. When in doubt, a third reviewer $(\mathrm{CvP})$ will be consulted for the final decision. The same two reviewers will conduct the full-text screening, which again will be pilot tested on a random sample of articles. No formal quality assessment will be performed.

\section{Stage 4: charting the data}

One reviewer (CR-K) will extract the data using a descriptive charting table designed for this review. The charting table will be pretested in collaboration with the second reviewer (AN) on a minimum of five articles to ensure consistency of data extraction. At this stage, the charting 
table may be further refined if necessary. The following key information will be extracted:

- author(s)

- year of publication

- publication type (eg, original research, report)

- study design

- population characteristic (eg, patient, citizen, ethnicity, sex, age, morbidity)

- provider characteristic (eg, profession)

- concept described (eg, coproduction, patient involvement)

- context (eg, country, healthcare setting, community setting)

- intervention (eg, goal setting)

- key findings (eg, factors influencing the coproductive relationship between immigrants and service providers)

\section{Stage 5: collating, summarising and reporting the results}

The scoping review will give an overview of a relatively broad field of literature, including a wide range of different publication types. Therefore, special attention will be paid to how the large amount of data will be presented. A guideline specifically for reporting scoping reviews is currently being developed by a group of researchers at the University of Toronto, but it has not yet been published. ${ }^{40}$ We will make efforts to secure use of this new guidance, but failing its availability, we will use a modified version of the Preferred Reporting Items for Systematic Reviews and Meta-Analysis (PRISMA) ${ }^{41}$ PRISMA items not appropriate for the purpose of this scoping review (eg, risk of bias) will be left out. The extracted data will be presented in tabular or diagrammatic form to give an overview of the amount, type and distribution of included literature. We will use NVivo software (V.11, QSR International) for coding and analysing the literature.

The plan for data presentation and discussion is based on the three research questions for this scoping review. We expect to outline individual and context-related factors that influence coproduction processes between immigrants and their service providers. In addition, we will analyse how the identified factors affect the respective coproduction process. Despite the contextual and setting related differences between a community and a healthcare setting, we want to investigate whether the mechanisms behind coproduction in a community setting can be used for learning and as a source of inspiration for the healthcare sector.

\section{IMPLICATIONS}

Interest in understanding the needs of new and diverse groups of patients and creating health service that can meet these needs in an interdependent, patient-centred way invites a new approach. 'Coproduction' may open up new perspectives and possibilities to improve the contribution of healthcare service to health. Findings of this study will provide an innovative perspective on

\section{Box 1 Inclusion/exclusion criteria}

Inclusion criteria:

- Languages: published in English, Danish, Swedish, Norwegian or German languages.

- Date: published from 2007 and onwards.

- Peer-reviewed literature: any study design and methodology.

- Non-peer-reviewed literature: reports, government documents, guidelines, policy papers and dissertations.

- Population: Immigrants defined as foreign-born people who have moved to another country for the purpose of settlement, as well as ethnic minority patients or citizens whose ethnicity and/or cultural traditions differ from those of the main population who coproduce services with healthcare/social providers or researchers.

- Coproduction as method: publications focusing on the joint creation of value for the coproducers through, for instance, the development, implementation, or evaluation of interventions, self-management plans, services, tools or knowledge.

- Coproduction as outcome: publications that report on planned/ unexpected coproduced outputs and outcomes, even if not initially planned.

- The coproducers both contribute resources and benefit from the value created by the service provided.

- Publications that report on concrete improvement strategies for explicit collaboration or coproduction between ethnic minorities and service providers.

Setting: coproduction in healthcare or social/community settings.

\section{Exclusion criteria:}

- Publications on how to involve and increase immigrant participation in research, trials or screening interventions (unless they were directly involved in the development and design of these interventions and directly benefited from the value created through the research).

- Publications focusing on coproduction involving only more resourceful representatives of the immigrant target group.

- Publications focusing purely on consulting immigrants or service providers on their perspectives and opinions.

- Publications focusing on the recruitment/education of voluntary community (health) workers.

- Publications focusing only on the consequences of suboptimal immigrant-provider relationships.

- Publications on coproduction on organisation level (eg, between hospital departments, with private organisations).

coproduced healthcare service by immigrant patients and care providers in Danish healthcare. This study represents the first step of a research programme designed to develop a model of coproduction of healthcare service with immigrant patients. Such a model will be based on principles that can be useful in designing and evaluating patient-centred healthcare services for immigrant patient groups, not only in a Danish context but potentially in any setting where immigrant patients or other minority patient groups meet their care providers. A timeline for the entire scoping review process is presented in table 1.

\section{ETHICS AND DISSEMINATION}

This scoping review will include exclusively published data, gathered through searching the literature in electronic databases and other online sources. Thus, no 
Table 1 Timeline for protocol and scoping review

\begin{tabular}{|c|c|c|c|c|c|c|c|c|c|}
\hline & \multicolumn{7}{|l|}{2017} & \multicolumn{2}{|l|}{2018} \\
\hline & June & July & August & September & October & November & December & January & February \\
\hline \multicolumn{10}{|l|}{$\begin{array}{l}\text { Writing } \\
\text { protocol }\end{array}$} \\
\hline \multicolumn{10}{|l|}{ Search } \\
\hline \multicolumn{10}{|l|}{ Screening } \\
\hline
\end{tabular}

ethics committee approval is required for this study. The protocol will support a systematic and transparent process of preparing and conducting the entire review process. The results will be disseminated through presentations at national and international clinical conferences and relevant seminars and networks on coproduction and/or immigrant health to relevant stakeholders and immigrant patient groups, and will be published in a peer-reviewed journal.

Acknowledgements The authors thank information specialist Line Bruun Hansen from the University Library at University of Southern Denmark, campus Esbjerg and librarian Berit Alving from the Videncentret at Odense University Hospital for their expertise provided in guiding the design of the draft search strategy for this scoping review.

Contributors CR-K drafted the protocol. AN, PB and MS helped conceptualise the research, and reviewed and edited the protocol. CvP obtained funding, conceptualised the research and reviewed and edited the protocol.

Funding This study is financed by the Center for Quality in the Region of Southern Denmark and the Department of Regional Health Research at the University of Southern Denmark.

Competing interests None declared.

Patient consent Not required.

Provenance and peer review Not commissioned; externally peer reviewed.

Data sharing statement № additional data available.

Open Access This is an Open Access article distributed in accordance with the Creative Commons Attribution Non Commercial (CC BY-NC 4.0) license, which permits others to distribute, remix, adapt, build upon this work non-commercially, and license their derivative works on different terms, provided the original work is properly cited and the use is non-commercial. See: http://creativecommons.org/ licenses/by-nc/4.0/

(C) Article author(s) (or their employer(s) unless otherwise stated in the text of the article) 2018. All rights reserved. No commercial use is permitted unless otherwise expressly granted.

\section{REFERENCES}

1. Woodgate RL, Busolo DS, Crockett M, et al. A qualitative study on African immigrant and refugee families' experiences of accessing primary health care services in Manitoba, Canada: it's not easy!. Int J Equity Health 2017;16:5

2. Suurmond J, Uiters E, de Bruijne MC, et al. Negative health care experiences of immigrant patients: a qualitative study. BMC Health Serv Res 2011;11:10.

3. Ahmed S, See S, Shommu N, et al. Experiences of communication barriers between physicians and immigrant patients: A systematic review and thematic synthesis. Patient Experience Journal 2017:4:122-40.

4. Burgess DJ, Fu SS, van Ryn M. Why do providers contribute to disparities and what can be done about it? J Gen Intern Med 2004;19:1154-9.
5. Sodemann $\mathrm{M}$, Kristensen TR, Sångren $\mathrm{H}$, et al. [Barriers to communication between clinicians and immigrants]. Ugeskr Laeger 2015;177.

6. Derose KP, Escarce JJ, Lurie N. Immigrants and health care: sources of vulnerability. Health Aff 2007;26:1258-68.

7. Fiscella K, Epstein RM. So much to do, so little time: care for the socially disadvantaged and the 15-minute visit. Arch Intern Med 2008;168:1843-52

8. Coulter A, Ellins J. Patient-focused interventions: a review of the evidence. London, UK: The Health Foundation, 2006.

9. Wagner EH, Austin BT, Davis C, et al. Improving chronic illness care: translating evidence into action. Health Aff 2001;20:64-78.

10. Ministry of Health. Nationalt Kvalitetsprogram for Sundhedsområdet 2015-2018. Copenhagen, DK, 2015.

11. Saha S, Beach MC, Cooper LA. Patient centeredness, cultural competence and healthcare quality. J Natl Med Assoc 2008;100:1275-85.

12. Street RL, Gordon HS, Ward MM, et al. Patient participation in medical consultations: why some patients are more involved than others. Med Care 2005;43:960-9.

13. Millenson ML. When "patient centred" is no longer enough: the challenge of collaborative health: an essay by Michael L Millenson. BMJ 2017;358:j3048.

14. Shay LA, Lafata JE. Where is the evidence? A systematic review of shared decision making and patient outcomes. Med Decis Making 2015;35:114-31.

15. Batalden M, Batalden $\mathrm{P}$, Margolis $\mathrm{P}$, et al. Coproduction of healthcare service. BMJ Qual Saf 2016;25:509-17.

16. Ostrom E. Crossing the great divide: coproduction, synergy, and development. World Dev 1996;24:1073-87.

17. Palumbo R. Contextualizing co-production of health care: a systematic literature review. Int J Public Sector Manage 2016;29:72-90.

18. Voorberg WH, Bekkers VJJM, Tummers LG. A systematic review of co-creation and co-production: embarking on the social innovation journey. Public Manage Rev 2015;17:1333-57.

19. Batalden P. A reflection on "Coproduction of healthcare service" (September 2017). New Hampshire, USA: The Dartmouth Institute, 2017.

20. Osborne SP, Radnor Z, Strokosch K. Co-production and the cocreation of value in public services: a suitable case for treatment? Public Manage Rev 2016;18:639-53.

21. Essén A, Värlander SW, Liljedal KT. Co-production in chronic care: exploitation and empowerment. Eur J Mark 2016;50:724-51.

22. Poulsen L. Embedsmandsrollen i borgerdrevet samskabelse - En ny kommunal embedsmandsrolle [The role of public officials in citizendriven partnership. Roskilde: Roskilde University, 2017.

23. Færch C. Borgerdrevet samskabelse i kommunerne Embedsmændenes nye rolle [Citizen-driven co-production in municipalities]. Roskilde: Roskilde University, 2017.

24. Jakobsen M, Andersen SC. Coproduction and equity in public service delivery. Public Adm Rev 2013;73:704-13.

25. Andersen LL, Espersen HH. Partnerskaber og samarbejder mellem det offentlige og civilsamfundet. [Partnerships and cooperation between the public sector and the civil society]. Copenhagen: Socialstyrelsen [The National Board of Social Services], 2017.

26. Tortzen A. Samskabelse i kommunale rammer - hvordan kan ledelse understøtte samskabelse [Coproduction in the municipal sector]. Roskilde: Roskilde University, 2016.

27. Turnaas S. The professional side of co-production. Tampere: University of Tampere, 2016.

28. Arksey H, O'Malley L. Scoping studies: towards a methodological framework. Int J Soc Res Methodol 2005;8:19-32. 
29. Levac D, Colquhoun H, O'Brien KK. Scoping studies: advancing the methodology. Implement Sci 2010;5:69.

30. Peters MDJ, Godfrey CM, Mclnerney P, et al. The Joanna Briggs Institute Reviewers' Manual 2015: Methodology for JBI Scoping Reviews. Adelaide: The Joanna Briggs Institute, 2015.

31. Bate P. Context is everything. In: Perspectives on context. London: The Health Foundation, 2014.

32. Laine C, Davidoff F. Patient-centered medicine. A professional evolution. JAMA 1996;275:152-6.

33. Loeffler E, Power G, Bovaird T, et al. Co-production of health and wellbeing in Scotland. Birmingham, UK: Governance International, 2013.

34. Perruchoud R, Redpath-Cross J. Glossary on migration. book glossary on migration. Geneva: International Organisation for Migration (IOM), 2011.

35. Esholdt $\mathrm{H}$, Fuglsang M. Etniske forskelle i patienters oplevelser [Ethnic differences in patient experiences]. Region Hovedstaden: Enheden for Brugerundersøgelser, 2009.
36. Jervelund SS, Malik S, Ahlmark N, et al. Morbidity, self-perceived health and mortality among non-western immigrants and their descendants in denmark in a life phase perspective. $J$ Immigr Minor Health 2017;19:448-76.

37. Oxford Dictionaries. Definition of "ethnic minority" in English. Oxford, UK: Oxford University Press.

38. Alford J. Engaging public sector clients: from service-delivery to coproduction. New York: Palgrave Macmillan, 2009.

39. Bahle T. The changing institutionalization of social services in England and Wales, France and Germany: is the welfare state on the retreat? J Eur Soc Policy 2003;13:5-20.

40. Colquhoun $\mathrm{HL}$, Levac D, O'Brien KK, et al. Scoping reviews: time for clarity in definition, methods, and reporting. $J$ Clin Epidemiol 2014;67:1291-4.

41. Moher D, Liberati A, Tetzlaff J, et al. Preferred reporting items for systematic reviews and meta-analyses: the PRISMA statement. PLOS Med 2009;6:e1000097. 